\title{
Solute Clustering and Grain Boundary Segregation in Extruded Dilute Mg-Gd Alloys
}

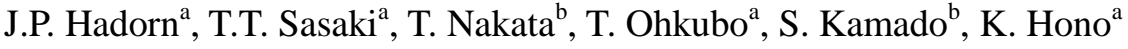 \\ ${ }^{a}$ National Institute for Materials Science, 1-2-1 Sengen, Tsukuba 305-0047, Japan \\ ${ }^{b}$ Department of Mechanical Engineering, Nagaoka University of Technology, 1603-1 \\ Kamitomioka-machi, Nagaoka 940-2188, Japan
}

\begin{abstract}
:
Two single-phase $\mathrm{Mg}$-Gd alloys (0.01 and 0.06 at\% Gd) were identically hot-deformed through an indirect extrusion process. High-angle annular dark field scanning transmission microscopy (HAADF-STEM) observations revealed that the Gd-richer alloy, which possessed a weaker texture, had substantial Gd-solute clustering and grain boundary segregation, while the more Gd-dilute alloy, which showed a markedly stronger texture, did not. It is suggested that clustering and grain boundary segregation play a role in the modification and consequential weakening of the overall texture.
\end{abstract}

Keywords: Mg alloy, HAADF-STEM, solute clustering, texture weakening 
Many commercial Mg alloys develop a strong crystallographic texture during hot-rolling and extrusion, which is attributed to its poor formability. However, microalloying rare-earth (RE) elements into $\mathrm{Mg}$ often causes the development of characteristically different texture components during hot-extrusion/rolling processing and/or subsequent annealing [1-5], which results in a significantly weaker texture overall. While particle-stimulated nucleation during dynamic recrystallization was first hypothesized as the mechanism for this phenomenon [1], its presence in dilute $\mathrm{Mg}$ - RE solid solution alloys indicates that second phases are not necessary to induce this texture weakening effect [5-7]. Solute can affect interfacial mobility through such means as grain boundary segregation and the formation of atomic clusters [8-9]. During hot-deformation, sufficient grain boundary drag can then alter the dynamic recrystallization (DRX) mechanism, which may consequently alter the final wrought texture [7].

For various Mg-RE systems, under a given processing condition, the deformation texture has been observed to change and ultimately weaken sharply with increasing RE composition. One binary Mg-RE system that has been extensively studied is Mg-Gd, which can undergo such a transition at solute concentrations in the single-phase region [5,10-11]. In fact, Gd shows one of the highest solubility of all RE elements in $\mathrm{Mg}$, being as high as 4.5 at\% at the eutectic temperature of $821 \mathrm{~K}$ (at 1 atm) [12-13]. The purpose of this study was to analyze the distribution of Gd solute within two extruded Mg-Gd alloys: a richer composition of 0.06 at\% Gd that shows a texture weakening effect and a dilute composition of 0.01 at\% Gd that does not under the same extrusion conditions. More specifically, a comparative study was performed to determine if there is a correlation between this observed texture modification/weakening ability and the presence of mobility inhibiting features such as solute clustering and grain boundary solute segregation. A recent atom probe tomography (APT) study [10] has indicated the presence of Gd clustering within the matrix of an extruded Mg- 0.24 at\% Gd alloy; however, microscopic characterization and verification is necessary to determine the nature of these clusters, especially whether they exist as solute within the hcp $\alpha-\mathrm{Mg}$ matrix phase or as nanoscale secondary phase precipitates. 
By employing high-angle annular dark field scanning transmission microscopy (HAADF-STEM), Gd solute within the Mg matrix can be observed through Z-contrast. This imaging technique has recently been successfully used to detect periodic segregation of individual Gd atoms to twin boundaries in deformed Mg-Gd and Mg-Gd-Zn-Zr alloys [14]. To select one strongly textured and one weakly textured Mg-Gd alloy suitable for this comparative study, the compositions and extrusion conditions were chosen based on the results of relevant texture investigations reported by Stanford [11].

For this study, two extruded Mg-Gd alloys, containing 0.01 and 0.06 at\% Gd (afterwards referred to as $0.01 \mathrm{Gd}$ and $0.06 \mathrm{Gd}$, respectively), were prepared. The alloys were first produced in an electric furnace, and then cast into a cylindrical mold. The cooled ingots were solutionized at $798 \mathrm{~K}$ for $24 \mathrm{~h}$ and then indirectly extruded at $723 \mathrm{~K}$ at a rate of $1.6 \mathrm{~mm} / \mathrm{s}$ using a circular die with an extrusion ratio of $\mathrm{R}=20$. Electron backscattered diffraction (EBSD) analysis was used to analyze the microstructures and crystallographic textures of the extruded alloys. Representative inverse pole figure (IPF) maps were obtained using a Carl Zeiss CrossBeam 1540EsB field emission scanning electron microscope (SEM) equipped with an EBSD detector. An FEI Titan transmission electron microscope (TEM) equipped with an aberration probe corrector was used to study the distribution of Gd solute within the $\alpha-\mathrm{Mg}$ matrix. Due to the large difference in atomic number between $\mathrm{Mg}(\mathrm{Z}=12)$ and $\mathrm{Gd}(\mathrm{Z}$ $=64$ ), the clustering and segregation of Gd atoms were observed using HAADF-STEM imaging, since the large $\mathrm{Z}^{2}$ ratio $(\approx 28.4)$ provided large atomic contrast. To distinguish between solute and Gd-rich nanoscale precipitates, it was necessary to achieve atomic resolution. Therefore, in this investigation, hcp $\alpha-\mathrm{Mg}$ grains were oriented along favorable zone axes that easily allowed the crystallographic structure of the atoms to be distinguished. When imaging grain boundaries, where both grains have different orientations, the sample was tilted so that one of the grains was oriented along such a crystallographic direction, as this was enough to categorize the entire interfacial region. Finally, electron energy loss spectrometry (EELS) analysis was used in cases where comparison of regions of similar sample thickness was necessary. 
Under the present extrusion conditions, a texture transition occurs with increasing Gd composition from 0.01 to 0.06 at.\% Gd. Figure 1 shows EBSD IPF maps taken along representative transverse sections of the 0.01 and $0.06 \mathrm{Gd}$ extruded alloys, along with their corresponding IPFs. The 0.01Gd alloy shows a stronger texture with a $[10 \overline{1} 0]$ component aligned along the extrusion direction, typical to what is generally observed for extruded conventional alloys. However, in the previous work on Mg-Gd alloys by Stanford [11], while the strongest texture was observed for the most dilute composition, 0.015 at.\% $\mathrm{Gd}$, the extrusion texture was reported to be similar to that of pure $\mathrm{Mg}$, specifically one where the crystallographic orientation along the extrusion direction is represented in the corresponding IPF by a band tilted from [hki0] [11,15]. The richer $0.06 \mathrm{Gd}$ alloy shows a different and significantly weaker texture with a $[\overline{1} 2 \overline{1} l]$ component near to but slightly tilted from [1 $2 \overline{1} 0]$, similar to the result reported by Stanford [11] for this composition and, in general, one commonly associated with extruded Mg-RE alloys [5,16-17]. This is commonly referred to as the "rare-earth," or "RE" texture in the literature. Therefore, consistent with the previous report, within this composition range, the Mg-RE, with increasing composition, undergo a texture modification to the RE texture, which is ultimately significantly weaker. Finally, the IPF maps in Figure 1 indicate that $0.06 \mathrm{Gd}$ has a larger grain size than does $0.01 \mathrm{Gd}$, and for $0.01 \mathrm{Gd}$, sparse twinning is observable along this imaging plane.

Figure 2 shows low magnification HAADF-STEM images comparing two representative edge-on grain boundaries in both extruded samples. In each case, the grain on the right is aligned along a $<11 \overline{2} 0>$ zone axis while the grain on the left is oriented along a much higher index zone axis. In each image, the slightly brighter appearance of the right grain compared to the left grain is a result of diffraction contrast, as evident by the presence of lattice fringes. The $0.06 \mathrm{Gd}$ alloy shows strong bright Z-contrast near the grain boundary due to Gd enrichment. Comparatively, the grain boundary in the $0.01 \mathrm{Gd}$ alloy shows little to no Z-contrast. Therefore, while $0.06 \mathrm{Gd}$ shows marked grain boundary segregation of $\mathrm{Gd}$, the more dilute alloy, $0.01 \mathrm{Gd}$, shows no feature of $\mathrm{Gd}$ segregation. 
Higher magnification HAADF-STEM images of both grain boundaries are shown in Figures 3a-b. At this scale, atomic resolution within the $<11 \overline{2} 0>$ oriented (right) grains is observed. Along this zone axis, the arrangement of the spots, corresponding to atomic columns, reveal the $\mathrm{ABAB}$ stacking present in the hcp $\alpha-\mathrm{Mg}$ matrix. Near the grain boundary in the $0.06 \mathrm{Gd}$ extruded alloy, the brighter spots, indicating a comparably higher fraction of Gd atoms, do not deviate from the matrix array. Also, no interference fringes, which would result from the presence of a different crystal structure, are observed. These results therefore suggest that the segregated Gd atoms are in solution with the matrix, as opposed to second phase grain boundary precipitates. Additionally, these Gd atoms do not appear uniformly segregated along the grain boundaries. Instead, they appear as an array of globs. This may indicate not only the segregation of Gd solute atoms but also Gd solute clusters.

The $\mathrm{Gd}$ solute distribution within the $\alpha-\mathrm{Mg}$ matrix of the $0.01 \mathrm{Gd}$ and $0.06 \mathrm{Gd}$ alloys is revealed in Figures 3c-d, which show comparative representative regions of both alloys taken at a similar sample thickness as determined by EELS spectra analysis; in both cases, the grains were imaged along the [0001] zone axis. The larger propensity of "bright" atoms in 0.06Gd indicates that this richer alloy has a higher matrix concentration of $\mathrm{Gd}$ solute atoms than does $0.01 \mathrm{Gd}$, as anticipated. Significant solute clustering is ubiquitously observed in $0.06 \mathrm{Gd}$, but not in $0.01 \mathrm{Gd}$ (although rare examples were found). However, for $0.01 \mathrm{Gd}$, grouping of $\mathrm{Gd}$ solute atoms is present in the form of pairs. Figure 4 shows two images of the same 0.01Gd matrix region, along [0001], taken at different defocus levels. For the defocus image shown on the left, two Gd atoms "appear" adjacent to each other. At different defocus levels, like the one shown on the right, both atoms simultaneously disappear, thus indicating that they are at essentially the same depth and therefore couplets. Such solute pairs are ubiquitously observed, and larger groups, like triplets and quadruplets, are sparsely present as well. Gd pairing is also present in the $0.06 \mathrm{Gd}$ matrix, in addition to clusters.

In the previous APT study on extruded Mg-0.24 at\% Gd [10], the prevalence of solute clustering was reported to be highest at the lower extrusion temperature $(688 \mathrm{~K})$ and decreased with increasing temperatures up to $833 \mathrm{~K}$. The present study has confirmed the presence of solute clusters 
in extruded dilute Mg-Gd alloys with weakened texture. Specifically, this study revealed that the more weakly textured $0.06 \mathrm{Gd}$ extruded alloy shows marked Gd solute clustering while the more strongly textured $0.01 \mathrm{Gd}$ alloy does not. In addition, while strong grain boundary segregation of Gd solute occurs in $0.06 \mathrm{Gd}$, no such segregation is observed in $0.01 \mathrm{Gd}$; grain boundary segregation was also reported in the 0.24 at\% Gd APT study at extrusion temperatures where solute clustering was reported [10]. This difference in solute distribution may be linked with differences in shape and strength of texture that the alloys develop during the extrusion process.

Solute clustering and grain boundary segregation, as observed in the 0.06Gd extruded alloy, is expected to greatly hinder the mobility of grain boundaries and dislocations through the solute drag effect [8-9]. Discontinuous DRX is a common mechanism where recrystallized grains nucleate at high-angle grain boundaries; recrystallization is facilitated by the bulging and/or serration of the grain boundaries and therefore requires them to be mobile [18-19]. The orientation of these recrystallized grains are derived from surrounding existing grains and therefore retain the deformation texture, which for $\mathrm{Mg}$ alloys, is often highly textured. As a result, discontinuous DRX is not expected to favor significantly weak texture in this case.

If grain boundary drag is sufficient, i.e. through solute drag in the case of the present work, discontinuous DRX can be suppressed, thereby promoting less mobile DRX. One such mechanism is continuous DRX, in which grains nucleate through the formation and rotation of subgrains during deformation [8,20-22]. This may result in a recrystallization texture that is different and, in this case, weaker than the strong deformation texture. Past studies on hot-rolled binary Mg-RE alloys have revealed a positive correlation between the presence of drag due to solute/second phase particles and the propensity for texture weakening as a result of DRX [7,23]. A change in texture shape and strength may therefore be linked with a transition in DRX mode. Large Gd solute clusters within the matrix may act as obstacles for the motion of dislocations. Specifically, sufficient RE solute clustering can interact with basal <a> dislocations, predominant in conventional Mg alloys, and promote cross-slip [24-25], which can permit their rearrangement into cell and subgrain structures characteristic of 
continuous DRX [8]. Positive correlations between texture modification/weakening ability and non-basal dislocation content have been observed in hot-rolled single phase Mg-Y alloys [6-7]. However, this is not to say that the presence of Gd solute clustering and grain boundary segregation are the only factors contributing to the RE texture in the $0.06 \mathrm{Gd}$ extruded alloy.

In conclusion, two Mg-Gd alloys were hot-extruded: a Gd-richer composition, 0.06Gd, results in a weaker RE texture compared with a more Gd-dilute composition, $0.01 \mathrm{Gd}$, which shows a stronger texture typical of conventional Mg alloys. Aberration corrected STEM observations verified the presence of significant clustering and grain boundary segregation of Gd atoms in the 0.06Gd alloy. Hence, the change in texture is attributed to a transition in DRX mode due to the effect of Gd on the mobility of grain boundaries and dislocations. Although significant solute clustering was not abundant in the $0.01 \mathrm{Gd}$ alloy, Gd atoms within the matrix were ubiquitously observed as pairs.

This work was supported by JST, ALCA. 
Figure 1: Representative EBSD IPF maps of $0.01 \mathrm{Gd}$ and $0.06 \mathrm{Gd}$, each taken along the transverse plane; corresponding IPF also shown below each map.

Figure 2: Low magnification STEM images of edge-on grain boundaries comparing, through Z-contrast, grain boundary segregation of $\mathrm{Gd}$ in 0.01 and 0.06 at\% $\mathrm{Gd}$ alloys. In both cases, right grain is aligned along [112̄0] zone axis.

Figure 3: Comparative shear corrected HAADF-STEM images showing atomic resolution: a-b) higher magnification images of 0.01 and 0.06 at\% Gd grain boundaries, respectively, presented in Figure 2 (right grains aligned along [11 $\overline{2} 0]$ zone axis); c-d) comparative images of 0.01 at.\% Gd and 0.06 a\% Gd matrices, respectively at similar sample thicknesses ([0001] zone axis).

Figure 4: HAADF-STEM images of same matrix region in $0.01 \mathrm{a} \% \mathrm{Gd}$ taken at different defocus conditions to reveal Gd atom couplet ([0001] zone axis). 


\section{$\underline{\text { References: }}$}

[1] E.A. Ball, P.B. Prangnell, Scripta Metall. Mater. 31 (1994) 111-116.

[2] J.W. Senn, S.R. Agnew, in: M.O. Pekguleryuz, L.W.F. Mackenzie (Eds.), Proceedings of Magnesium Technology in the Global Age, (Montreal, Canada, 2006) 115-130.

[3] J. Bohlen, M.R. Nurnberg, J.W. Senn, D. Letzig, S.R. Agnew, Acta Mater. 55 (2007) 2101-2112.

[4] J.W. Senn, S.R. Agnew, in: H. I. Kaplan (Ed.), Magnesium Technology 2008: Proceedings of TMS, (TMS, Warrendale, PA, 2008) 153-158.

[5] N. Stanford, M.R. Barnett, Mat. Sci. Eng. A 496 (2008) 399-408.

[6] K. Hantzsche, J. Bohlen, J. Wendt, K.U. Kainer, S. Yi, D. Letzig, Scripta Mater. 63 (2010) 725-730.

[7] J.P. Hadorn, K. Hantzsche, S. Yi, J. Bohlen, D. Letzig, J.A. Wollmershauser, S.R. Agnew, Metall. Mater. Trans. A 43 (2012) 1347-1362.

[8] F.J. Humphreys, M. Hatherly, Recrystallization and Related Annealing Phenomena, 1st Ed., (Elsevier Science Inc., Atlanta, GA), 1995.

[9] E.A. Grey, G.T. Higgins, Acta Metall. 21 (1973) 309-321.

[10] N. Stanford, G. Sha, J.H. Xia, S.P. Ringer, M.R. Barnett, Scripta Mater. 65 (2011) 919-921.

[11] N. Stanford, Mat. Sci. Eng. A 527 (2010) 2669-2677.

[12] L.L. Rokhlin, Izv. Akad. Nauk SSSR, Met. (1) (1977) 181-183.

[13] L.L. Rokhlin, Magnesium Alloys Containing Rare Earth Metals, (Taylor and Francis, NY, 2003).

[14] J.F. Nie, Y.M. Zhu, J.Z. Liu, X.Y. Fang, Science 340 (2013) 957-960.

[15] R. Cottam, J. Robson, G. Lorimer, B. Davis, Mat. Sci. Eng. A 485 (2008) 375-382.

[16] L.W.F. Mackenzie, B. Davis, F.J. Humphreys, G.W. Lorimer, Mater. Sci. Tech. 23 (2007) 1173-1180.

[17] W.X. Xu, L. Jin, Z.Y. Zhang, W.J. Ding, J. Dong, J. Alloy. Compd. 585 (2014) 111-119.

[18] M.R. Barnett, Acta Mater. 55 (2007) 3271-3278.

[19] M. Guillope, J.P. Poirier, J. Geophys. Res. 84 (1979) 5557-5567.

[20] S.H. White, P. Roy. Soc. Lond. A Mat. 283 (1976) 69-86.

[21] S.E. Ion, F.J Humphreys, S.H. White, Acta Metall. Mater. 30 (1982) 1909-1919.

[22] P.G. Tungatt, F.J Humphreys, Acta Metall. Mater. 32 (1984) 1625-1635.

[23] J.P. Hadorn, K. Hantzsche, S. Yi, J. Bohlen, D. Letzig, S.R. Agnew, Metall. Mater. Trans. A 43 (2012) 1363-1375.

[24] A. Urakami, M.E. Fine, Scripta Metall. Mater. 4 (1970) 667-672.

[25] A. Sato, M. Meshii, Acta Metall. Mater. 21 (1973) 753-768. 


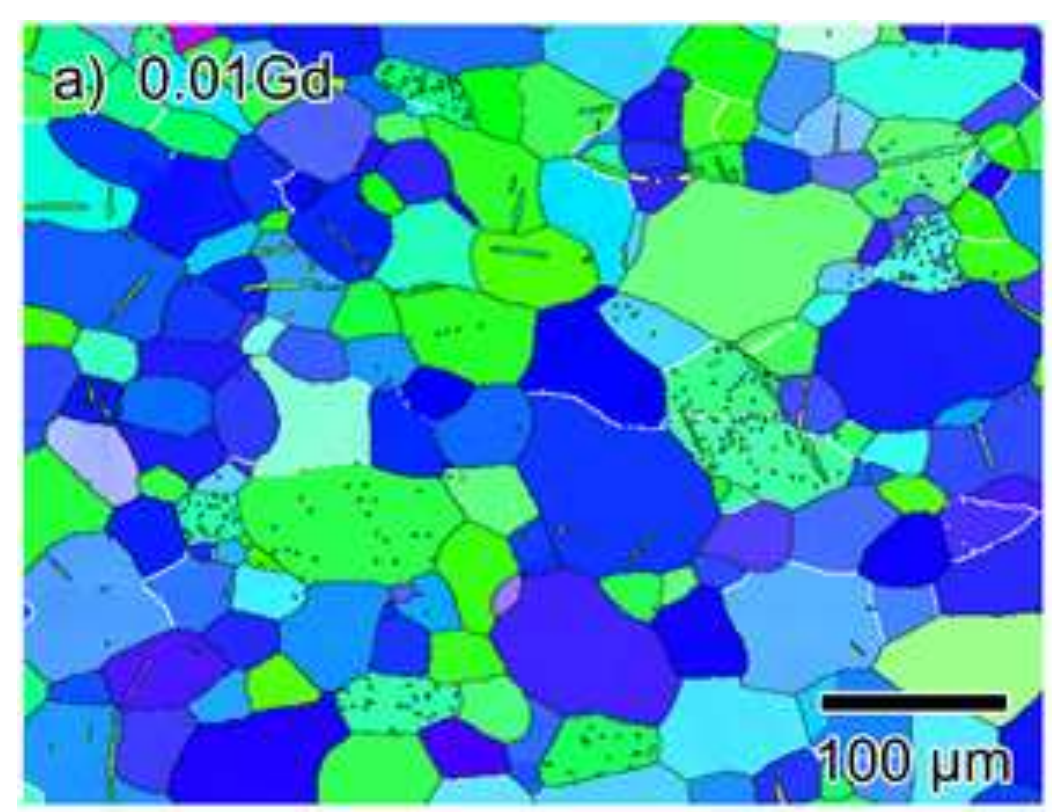

[0001]

[1ํํㅇ]

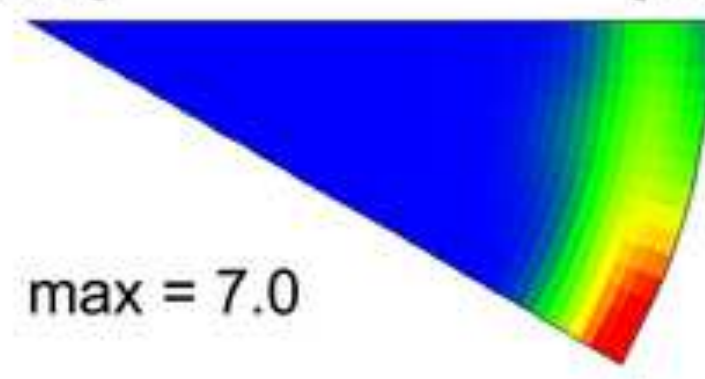

[0110]$$
\text { (0110] }
$$

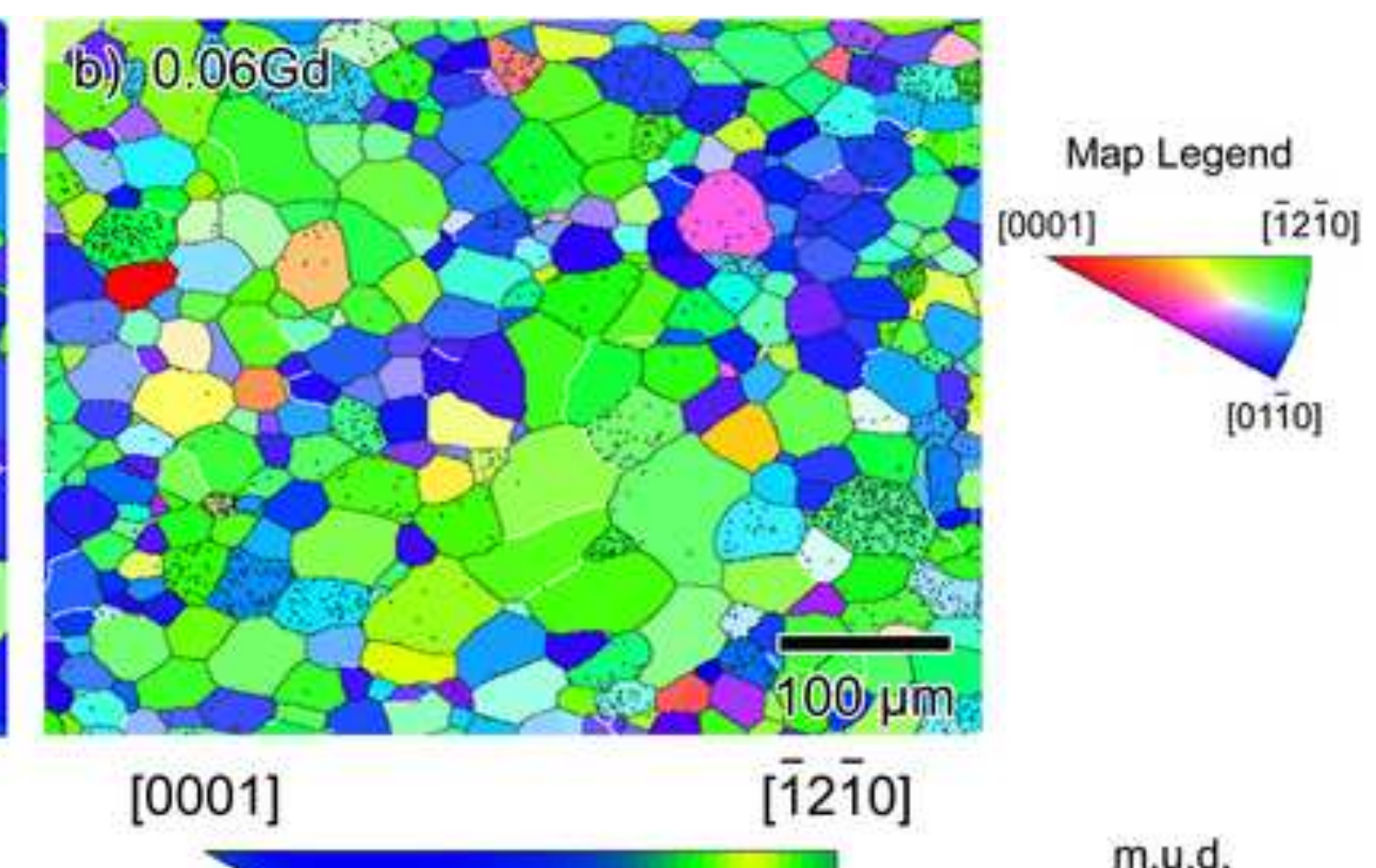

[12 10$]$

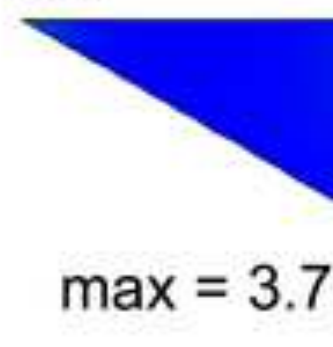

$\max =3.7$

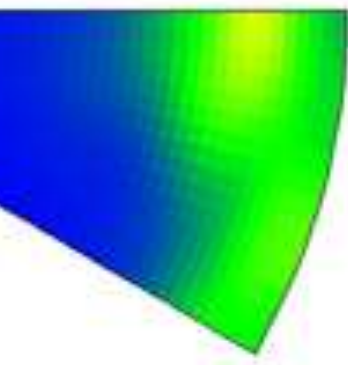

[01̄0]
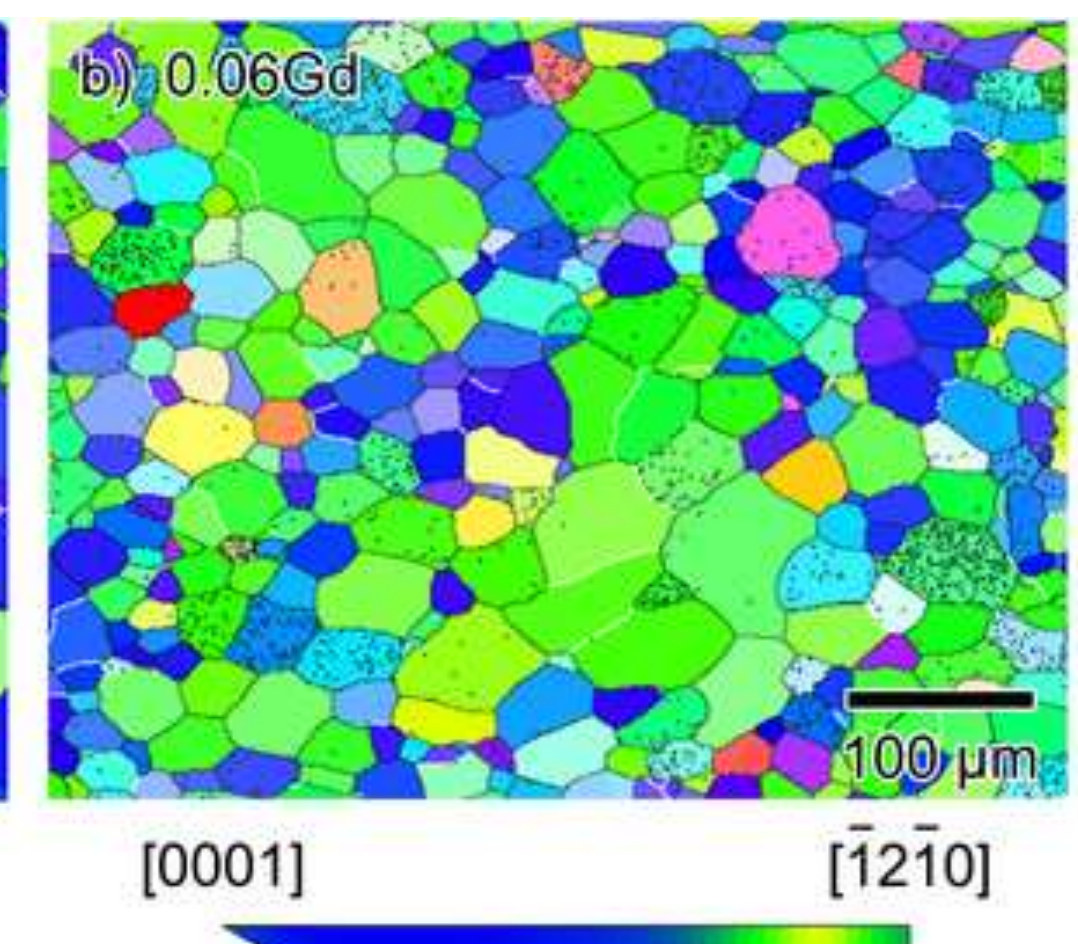

m.u.d.

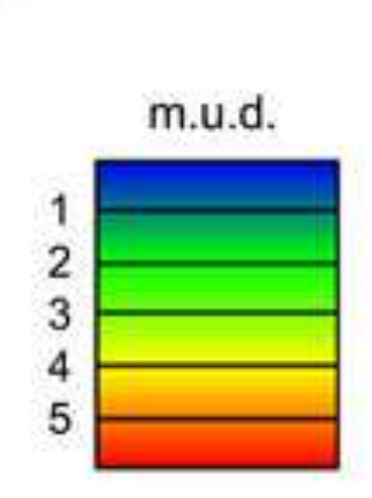

Map Legend

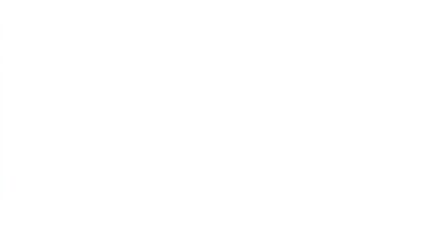


a)

$\mathrm{Mg}-0.01 \mathrm{Gd}$

\section{บิ \\ G.B.}

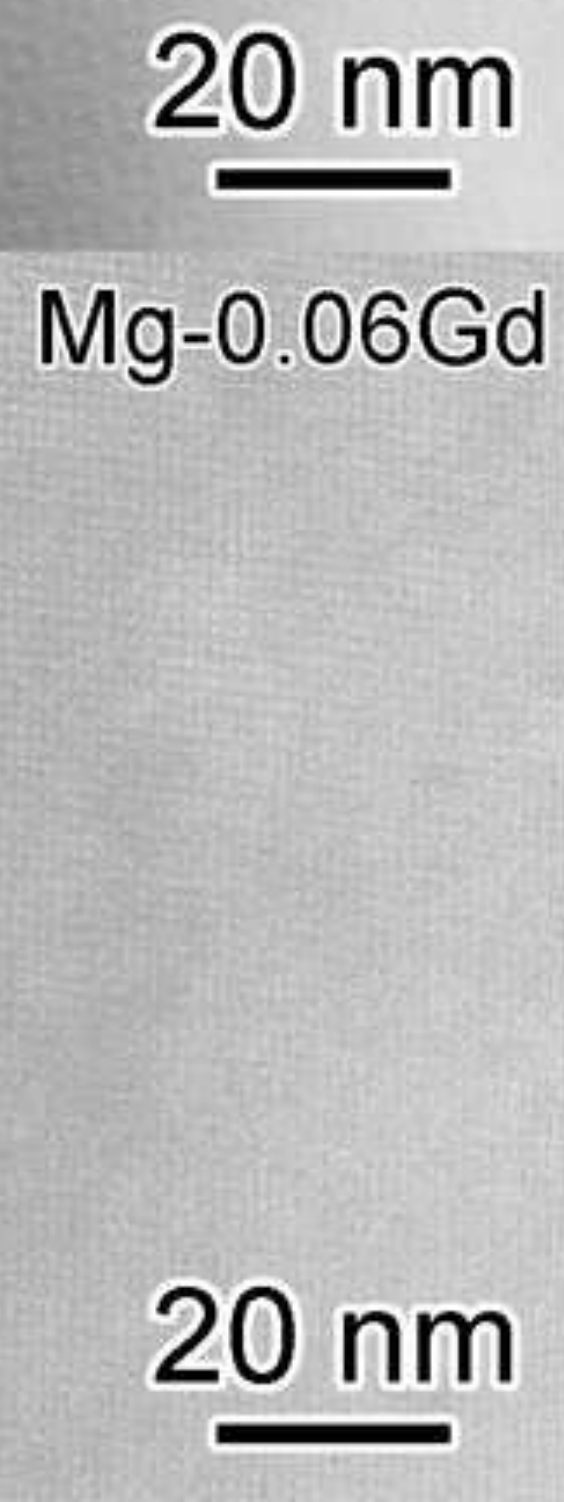

b)

$\mathrm{Mg}-0.06 \mathrm{Gd}$

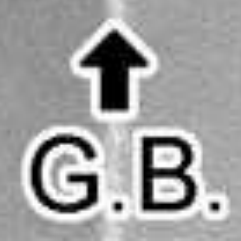




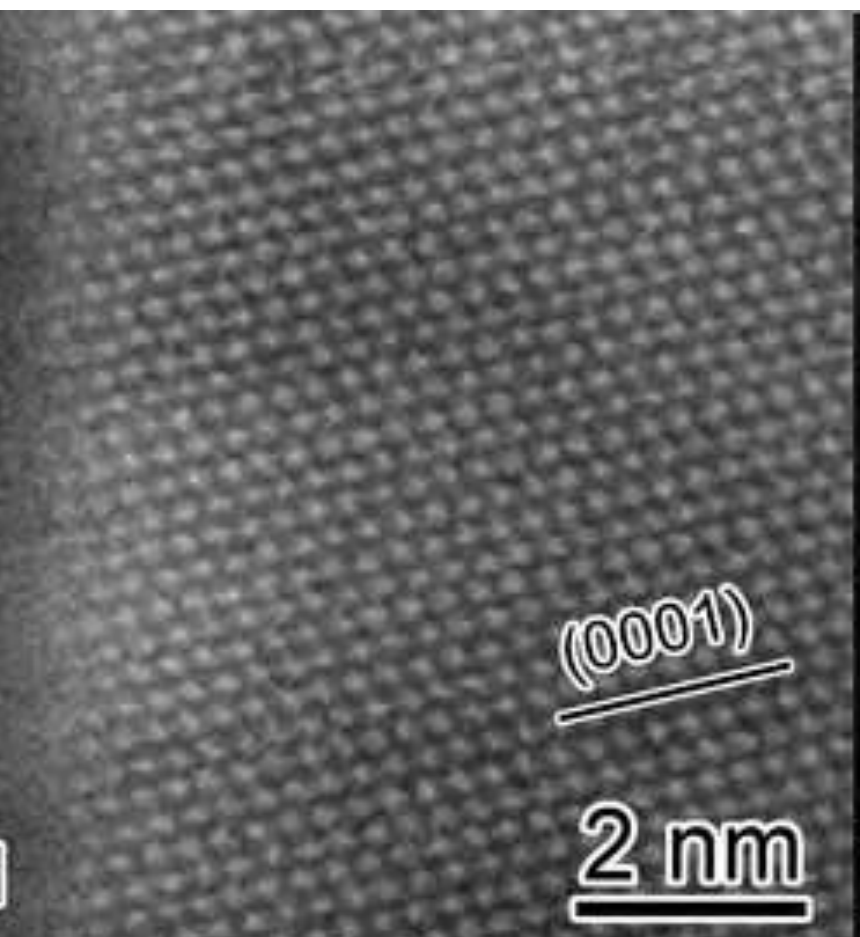

\section{b)}

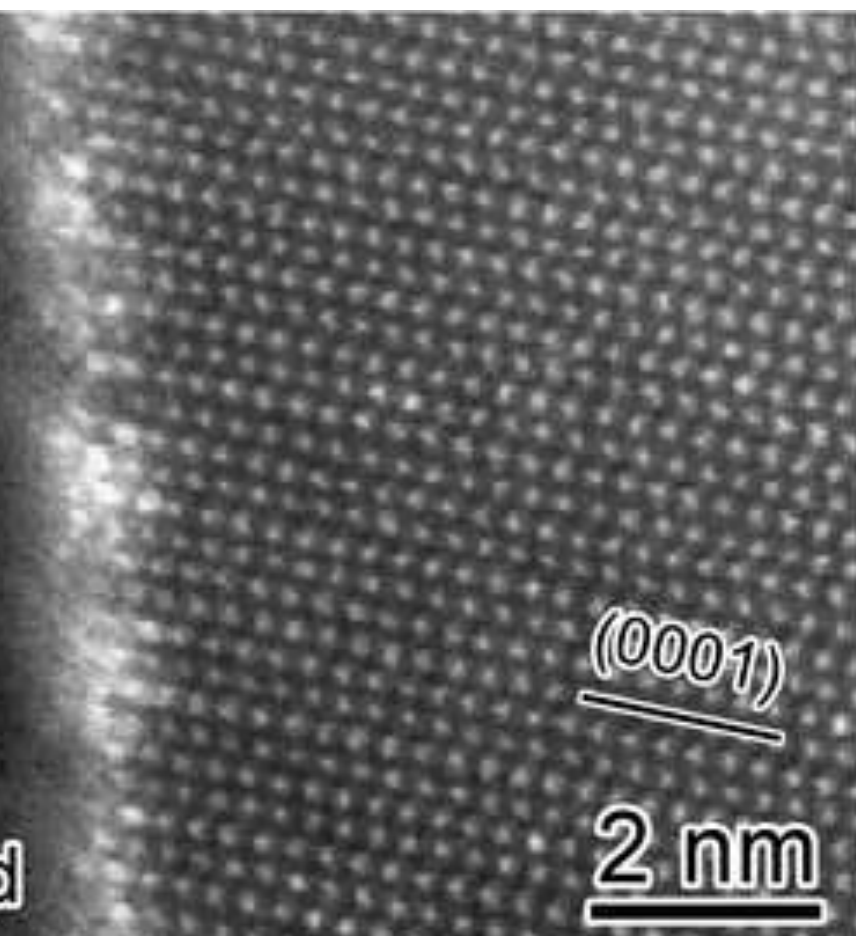

$\mathrm{Mg}=0.01 \mathrm{G} d$ c) $m$ W

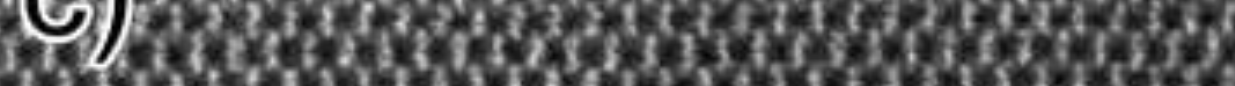
6. d) 8. Q.

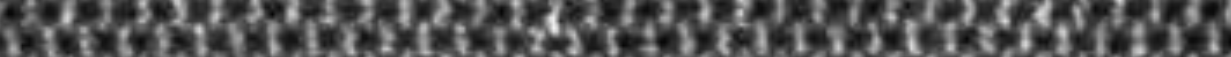
1.8.

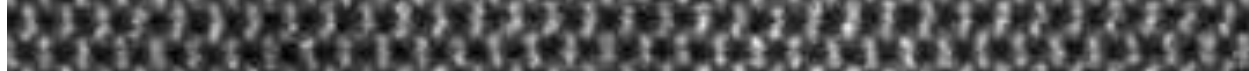

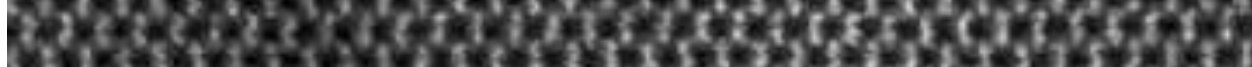

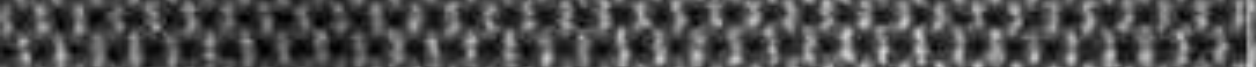

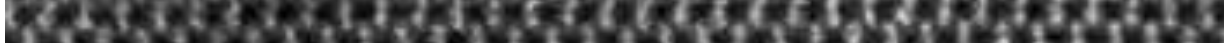

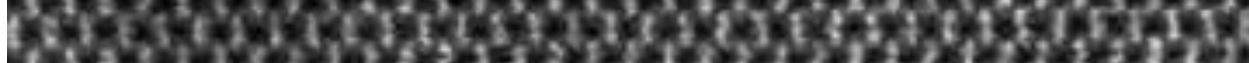

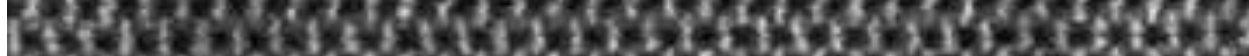

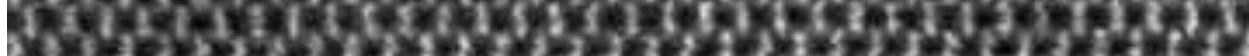

$M g=0.06 \mathrm{G} d$

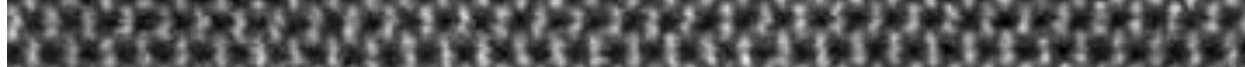
Mg:0iGd W (15) (1. (1. B

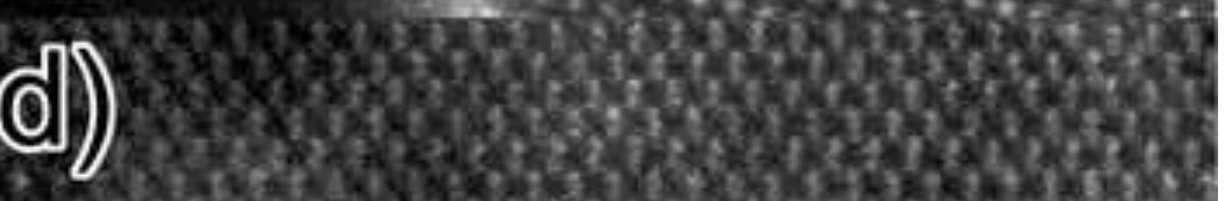


1. G Gd-couplet
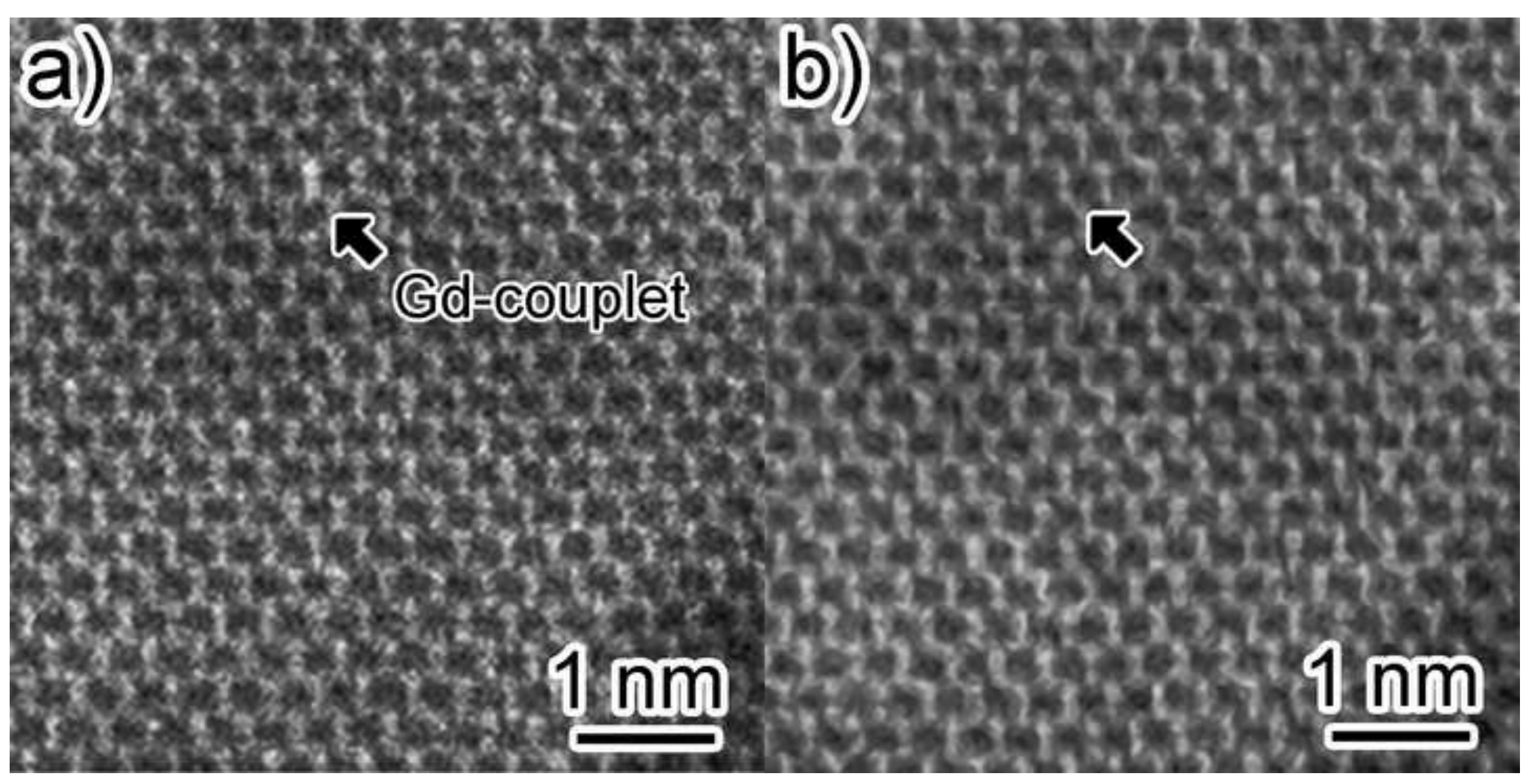

\section{b)}

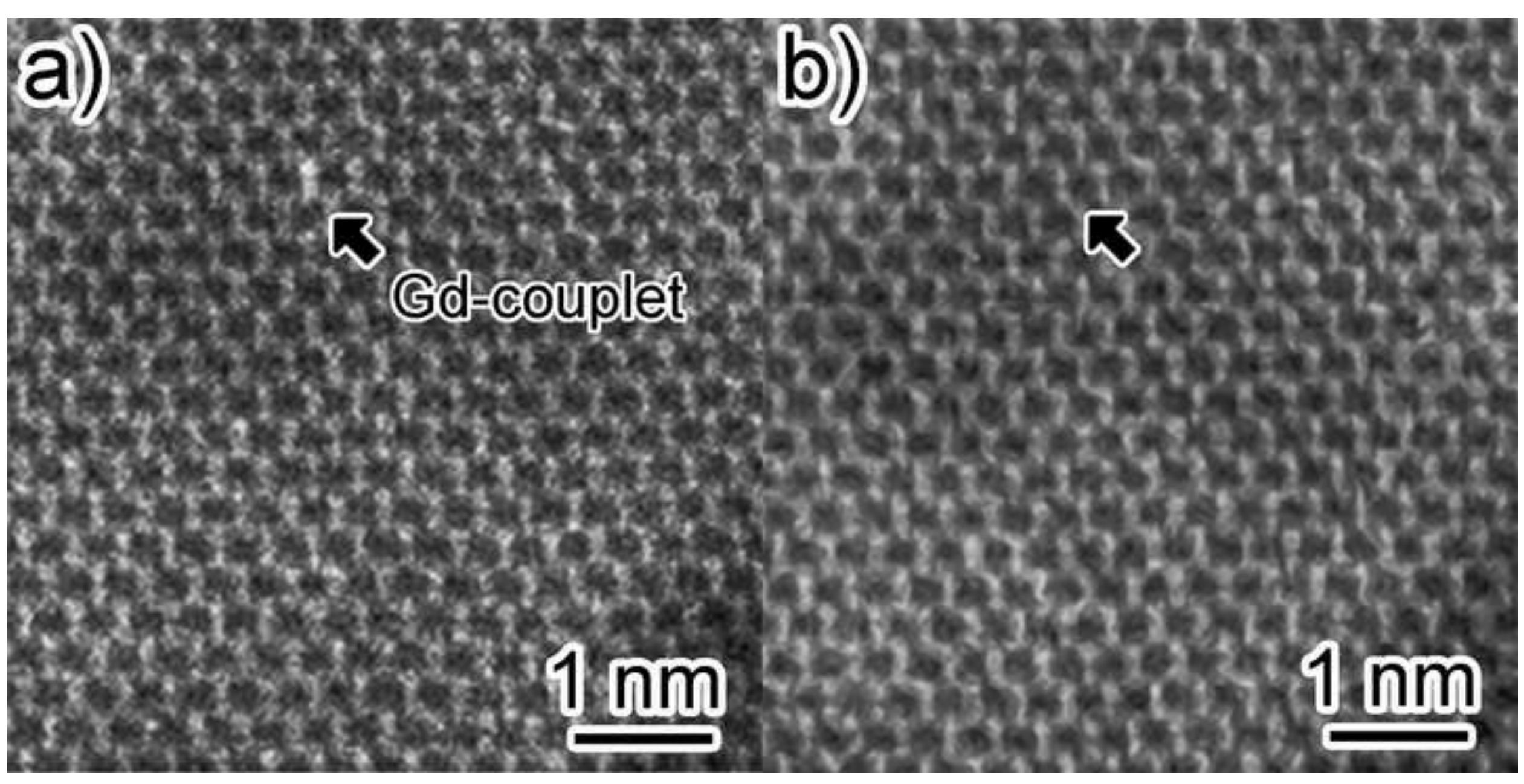
. 